\title{
Laboratory Experiments for Wave-Driven Sand Transport Prediction
}

\author{
T. O'Donoghue ${ }^{1}$ and D.A. van $\operatorname{der} \mathbf{A}^{2}$

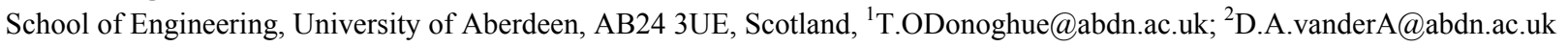

\begin{abstract}
Since the first NCK-Days 20 years ago, a significant body of large-scale laboratory experiments on wave-driven sand transport has been conducted in oscillatory flow tunnels and large wave flumes. The experiments have yielded measures of net sand transport rates for a wide range of flow and sand conditions and have provided insights that have informed the development of practical predictors for sand transport in oscillatory flows and under waves. An overview of these experiments and a commentary on some of the important insights and quantitative results is presented. Particular attention is given to unsteady aspects of the sand flux for ripple regime and fine sand sheet-flow conditions, the role of flow acceleration on bed shear stress and net transport, and the differences in net transport and transport processes occurring in tunnel oscillatory flows and occurring under progressive surface waves.
\end{abstract}

\section{INTRODUCTION}

Twenty years ago, the year of the first NCK-Days, Dibajnia and Watanabe [1992] published a formula for predicting sheet-flow sand transport under waves and currents. The formula uses a halfcycle approach whereby sand transported in each half-cycle of the near-bed oscillatory flow (Figure 1) is calculated based on a representative velocity for that half-cycle. The Dibajnia and Watanabe predictor is classed as "semi-unsteady" because it accounts for an unsteady (phase lag) effect whereby a proportion of sand entrained during one half-cycle remains in suspension and is transported during the following half-cycle. The half-cycle approach has since been adopted by others as the basis for more developed predictors [Dibajnia and Watanabe, 1996, 1998; Camenen and Larson, 2006; Silva et al., 2006; Van der Werf et al., 2007; Van der A et al., subm.]. Other practical formulae, not based on the half-cycle approach, have also been developed in the intervening years including Ribberink [1998], Drake and Calantoni [2001], Dohmen-Janssen et al. [2002], Soulsby and Damgaard [2005], Nielsen [2006], Van Rijn [2007], Wang [2007], Gonzalez-Rodriguez and Madsen [2007] and Suntoyo et al. [2008]. All of these predictors have the following in common: they comprise simple formulae making them readily useable within morphological models and they aim to capture essential processes within these formulae through parameterizations based on laboratory experiments.

The focus of this paper is on the laboratory experiments that have informed the development of the practical formulae for wave-driven sand transport. It presents an overview of the laboratory experiments of the last 20 years or so and indicates how insights and quantitative results from the experiments have been used to develop the predictors. The focus is on experiments conducted at large scale, by which we mean flows with period $T>$ $4 \mathrm{~s}$ and real sands (mostly in the range $0.12 \mathrm{~mm} \leq d_{50} \leq 0.54 \mathrm{~mm}$ ). Studies at this scale have been conducted in large wave flumes such as the GWK in Hannover and the Deltaflume in The Netherlands, and in large oscillatory flow tunnels such as the LOWT in The Netherlands, the AOFT in Aberdeen and the TOFT in Tokyo.

\section{MEASURED NET SAND TRANSPORT RATES}

Perhaps the most important contribution from experiments towards the development of practical predictors is the body of net sand transport rates measured for well-defined hydrodynamic and sand conditions, which can be used to calibrate and test the predictors. The transport rates are obtained by applying the conservation of mass principle to the measured pre- and post-test bed profiles and the volumes of sand collected from the ends of the test section at the end of an experiment. The same cannot be done in the field; indeed measurement of total net sand transport under waves is still not practically feasible in field conditions.

The database first described by Van der Werf et al. [2009] now contains 226 measured net transport rates for a wide range of fullscale conditions. Figure 2 presents the measured net transport rates

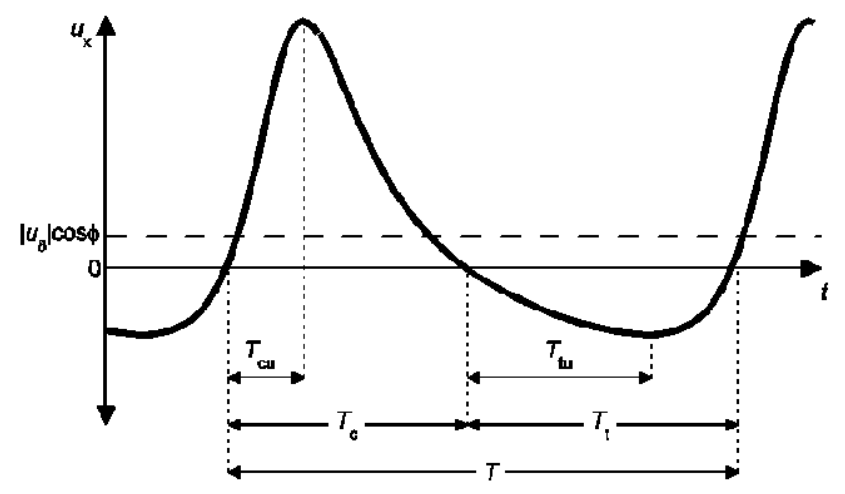

Figure 1. Definition sketch for near-bed velocity input to predictor; subscript "c" and " $t$ " refer to wave crest and trough respectively. $u_{\delta}$ is steady current at angle $\phi$ to wave direction. 


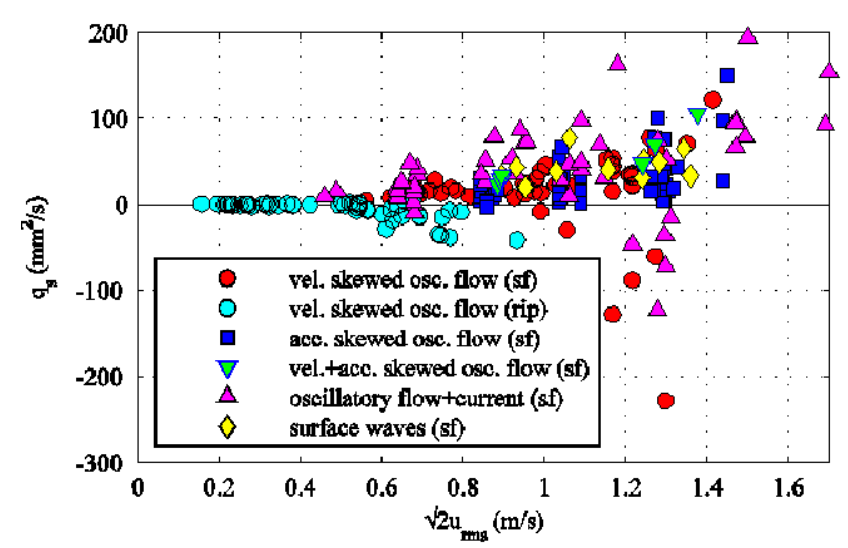

Figure 2. Measured net transport rates from large-scale laboratory experiments ( $s=$ sheet flow; rip = rippled beds).

plotted against $\sqrt{2} u_{\text {rms }}$, where $u_{\text {rms }}$ is rms free-stream horizontal orbital velocity. The results cover the ripple and sheet-flow regimes, progressive non-breaking surface waves, regular and irregular oscillatory flows with velocity and/or acceleration skewness and oscillatory flows with superimposed current. The range of net transport rates for given $\sqrt{2} u_{\text {rms }}$ in Figure 2 reflects the fact that transport rate depends on sand size, flow period and shape and whether the near-bed flow is generated by surface waves or is generated in an oscillatory flow tunnel. For oscillatory flows without superimposed current, it is the asymmetry in the flow kinematics between the positive and negative flow halfcycles that drives the transport: for velocity-skewed flow (circles in Figure 2), the asymmetry is asymmetry in velocity, with higher peak positive than peak negative velocity (a la Stokes-type wave); for acceleration-skewed flow (square symbols in Figure 2) the asymmetry is asymmetry in acceleration with higher peak positive than peak negative accelerations (sawtooth-type flow).

\section{RIPPLE REGIME}

A large number of full-scale laboratory experiments have been conducted over the past 20 years involving wave-generated sand ripples, both in wave flumes [Thorne et al., 2003; Williams et al., 2004; Cataña-Lopera and Garcia, 2006; O'Hara Murray et al., 2011] and in oscillatory flow tunnels [Ribberink and Al-Salem, 1994; Ahmed and Sato, 2001; O'Donoghue and Clubb, 2001; Sleath and Wallbridge, 2002; Van der Werf et al., 2006].

Primarily because of its impact on bed roughness, ripple geometry has been a main focus for many studies [Dumas et al., 2005; O'Donoghue et al., 2006; Pedocchi and Garcia, 2009]. Based on combined data from large-scale experiments covering a wide range of sand sizes, $2 \mathrm{D}$ and $3 \mathrm{D}$ ripples and regular and irregular flows, O'Donoghue et al. [2006] proposed the following Nielsen-type formulae for ripple height, $\eta$, and ripple length, $\lambda$ :

$$
\begin{gathered}
\frac{\eta}{a}=m_{\eta}\left\{0.275-0.022 \psi^{0.42}\right\} \\
\frac{\lambda}{a}=m_{\lambda}\left\{1.97-0.44 \psi^{0.21}\right\}
\end{gathered}
$$

where $\psi$ is mobility number and the multiplier $m$ for $\eta$ or $\lambda$ depends on sand size. Figure 3 shows the general good agreement between ripple dimensions calculated using Equations (1) and (2) and the corresponding measured ripple dimensions for the data used by O'Donoghue et al. [2006].

For practical sand transport predictors, ripple dimensions are important in quantifying the bed roughness for shear stress estimation and, for half-cycle-type formulations, determining the phase lag parameter which controls the degree of sharing of entrained sand between the present and subsequent flow halfcycle, as described below. Rippled bed roughness is commonly scaled to the ripple height, $k_{\mathrm{s}} \propto \eta$, or to the product of ripple height and steepness, $k_{\mathrm{s}} \propto \eta^{2} / \lambda$.

A number of large-scale experiments have studied the detailed sand transport processes occurring over ripples [Ribberink and AlSalem, 1994; Thorne et al., 2003; Van der Werf et al., 2006; O'Hara Murray et al., 2011]. Sediment transport over rippled beds has two components: a suspended component and a bedload component associated with ripple migration. During positive (onshore) flow, the onshore velocities transport sand along the stoss slope and over the ripple crest. Some of this sand is entrained in the developing lee vortex, which also entrains sand from the lee slope. The vortex increases in size as the flow slows, entraining more sand from the lee slope as it does so. The sand-laden vortex is then ejected into the main flow above the ripple at about the time of flow reversal, contributing to the negative-directed (offshore) suspended sediment transport. A proportion of the sand that has been carried up the stoss slope and over the ripple crest during the onshore flow does not get carried into suspension by the lee vortex. Instead it slumps down the onshore side contributing to onshore ripple migration. The same processes occur during the offshore flow half cycle. There is non-zero net transport if there is asymmetry between the two half-cycles, caused by asymmetry in the freestream flow. For example, for velocity-skewed oscillatory flow, the ripples are asymmetric, with steeper lee (onshore) slopes than stoss (offshore) slopes; the lee
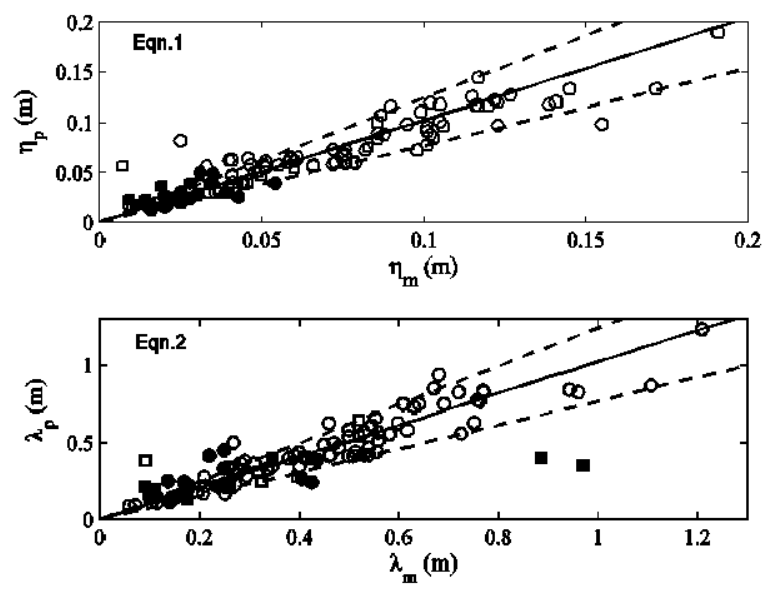

Figure 3. Ripple dimensions calculated using Equations (1) and (2) plotted against corresponding measured ripple dimensions; open/solid symbols correspond to $2 D / 3 D$ ripples and circles/squares correspond to regular/irregular flows [after O’Donoghue et al., 2006]. 
vortex is much stronger than the stoss vortex and more sand is ejected at the onshore-offshore flow reversal than at the offshoreonshore reversal, resulting in much lower onshore-directed suspended sand transport. At the same time, such flows cause onshore ripple migration. For velocity-skewed flow therefore, net suspended transport is offshore while net bedload transport is onshore and the net total transport (magnitude and direction) depends on the relative magnitudes of these two contributions.

The entrainment of sand during one half-cycle, its ejection into the flow at flow reversal and subsequent transport in the opposite direction during the following half-cycle, is a "phase lag" effect in the sense that the entrained sand concentration lags the flow velocity. Half-cycle transport predictors can account for this effect through a "phase lag parameter" which controls the proportion of sand entrained during one half-cycle that is transported in the following half-cycle. For example, in the "santoss" predictor [ Van $\operatorname{der} A$ et al., subm.], the phase lag parameter is

$$
P_{i}=\alpha \frac{\eta}{2\left(T_{i}-T_{i \mathrm{u}}\right) w_{s}}
$$

where $w_{\mathrm{s}}$ is sand settling velocity, the $T \mathrm{~s}$ are as indicated in Figure 1 with subscript $i$ being $c$ or $u$ for wave crest or trough respectively and $\alpha$ is a calibration parameter. The phase lag parameter represents the ratio of a representative "stirring height" and the sand settling distance within the decelerating phase of the flow half-cycle; for rippled bed, the stirring height is taken as proportional to ripple height. The higher the value of $P$, caused by higher ripples or a shorter flow deceleration phase, the greater the proportion of entrained sand that is carried over to the following half-cycle. Other predictors define phase lag parameters in a different way but with the same purpose of controlling the proportion of sand that is carried over to the following half-cycle [e.g. Van der Werf et al., 2006; Camenen and Larson, 2007].

\section{OSCILLATORY SHEET-FLOW}

Sheet flow occurs when wave-generated near-bed flow velocities are high, ripples are washed out and sand is transported in a water-sand mixture layer that is of order $\mathrm{cm}$ in thickness. Sheet-flow transport is considered a form of bed load, although textbook definitions of bed load or suspended load do not strictly apply. Based on laboratory experiments conducted in the LOWT [Ribberink and Al-Salem, 1994, 1995], Ribberink [1998] observed a near quasi-steady response in sheet flow sand concentration relative to the oscillating freestream flow velocity for sands with $d_{50}>0.2 \mathrm{~mm}$ and flows with period $T>4 \mathrm{~s}$. Observations of the fundamental processes, combined with measures of net sand transport for a range of conditions, led to Ribberink's quasi-steady bedload transport formula in which the instantaneous transport rate, $\phi(t)$, is directly related to the instantaneous (Shields) shear stress, $\theta(t)$, in a formula of the following kind:

$$
\phi(t)=m\left|\theta(t)^{n}\right| \frac{\theta(t)}{|\theta(t)|}
$$

where $m$ and $n$ are calibration coefficients. For velocity-skewed flows given by $u(t)=u_{1} \sin (\omega t)-u_{2} \cos (2 \omega t)$, with velocity skewness $R=\left(u_{1}+u_{2}\right) / 2 u_{1}$ and rms velocity $u_{\text {rms }}$, net transport based on Ribberink's quasi steady formula can be shown to be

$$
\phi_{N}=\frac{\alpha m}{\left[1+(2 R-1)^{2}\right]^{n}} \theta_{\sqrt{2} u_{\mathrm{rms}}}^{n}
$$

where $\alpha$ depends on $R$ and $n$ only. Figure 4 shows a comparison of Equation (5) $(R=0.63, n=1.65$ and $m=11)$ against measured (non-dimensional) net sand transport rates for velocity-skewed oscillatory flows with $R \approx 0.63$. For the coarser sands $\left(d_{50}>\right.$ $0.2 \mathrm{~mm}$ ), sheet-flow net transport rates are positive and increase with increasing Shields, and the Ribberink predictor is seen to agree well with the measured transport rates, supporting the validity of the quasi-steady approach for these coarser sands. However, the net transport results for the finer sands $\left(d_{50} \leq\right.$ $0.2 \mathrm{~mm}$ ) show a contrary behaviour: net transport rates are initially positive but become negative at higher Shields and show very high negative net transport at high Shields. For the finer sands therefore, the wave-generated sand transport process is not quasisteady: it is an unsteady process and the degree of unsteadiness depends on the flow condition and the sand size.

The unsteady behaviour seen in the net transport results for fine sand in Figure 4 is also seen in detailed process measurements for sheet flow conditions measured in the AOFT and LOWT oscillatory flow tunnels. Example measurements of the intra-wave erosion depth and sheet-flow layer thickness obtained via detailed concentration measurements within the sheet-flow layer are presented in Figure 5, which shows example results for three cases: (a) $0.27 \mathrm{~mm}$ sand in a velocity-skewed flow; (b) $0.13 \mathrm{~mm}$ sand in velocity-skewed flow; (c) $0.15 \mathrm{~mm}$ sand in accelerationskewed flow. In the case of Figure 5(a), we see a near quasi-steady behaviour in the time-dependent erosion depth and sheet-flow layer thickness for the medium sand. In contrast, we see strong unsteadiness in Figure 5(b) for the case of fine sand in the same velocity-skewed flow as in Figure 5(a). In this case, a proportion of sand entrained during each flow half-cycle does not settle back to the bed before flow reversal, and the proportion is greater for the positive flow half-cycle than for the negative flow half-cycle because positive velocity is higher and deceleration time is shorter; the result is deeper erosion and thicker sheet-flow layer thickness soon after the positive-negative flow reversal compared with after the negative-positive reversal.

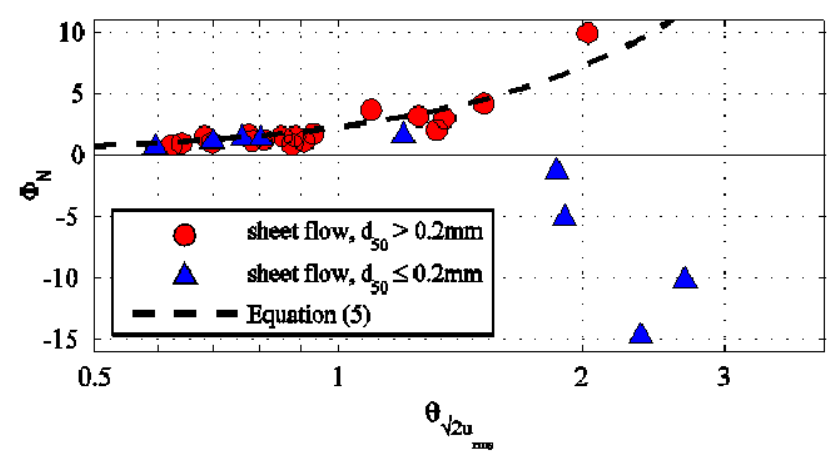

Figure 4. Measured net transport rates for sheet-flow, velocityskewed oscillatory flow with $R \approx 0.63$ and calculated net transport using Equation 4 with $m=11, n=1.65$ and $R=0.63$. 

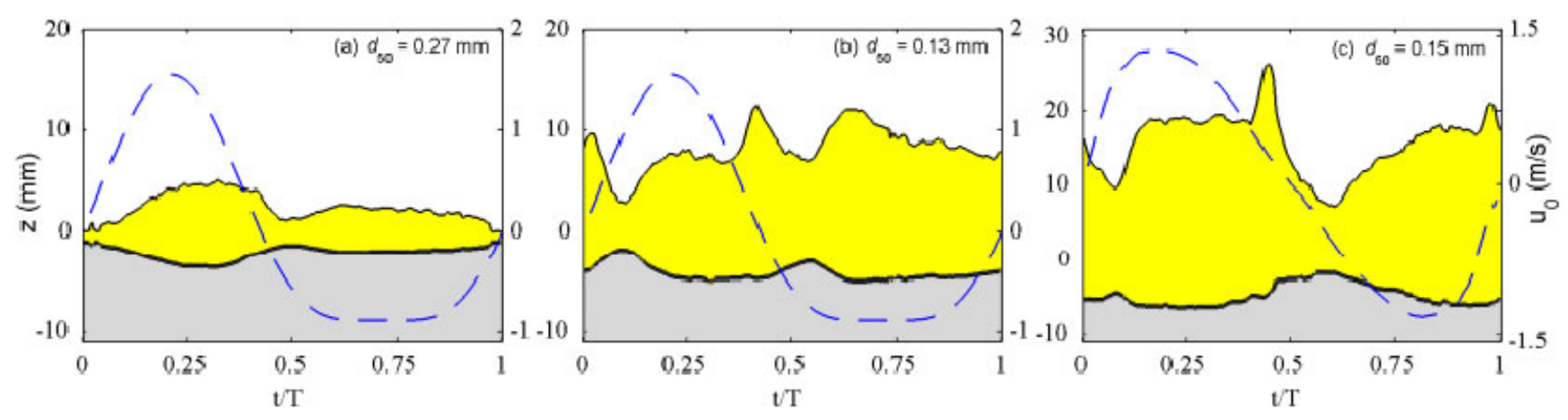

Figure 5. Measurements of erosion depth (black line) and sheet-flow layer thickness (shaded yellow) for (a) medium and (b) fine sand in a velocity-skewed flow and (c) for fine sand in acceleration-skewed flow; the free-stream velocity is indicated by the dashed line.

As for ripples, there is therefore a phase lag effect in sheet-flow transport processes, and the effect is stronger for finer sands and shorter period flows. For the santoss half-cycle-based predictor [Van der $A$ et al., subm.], a phase lag parameter for sheet flow is defined similarly to Equation (3), but with sheet flow layer thickness, $\delta_{\mathrm{s}}$, replacing ripple height as the representative stirring height. Recourse is again made to measurements from large-scale experiments to estimate $\delta_{\mathrm{s}}$. For example, Ribberink et al. [2008] propose $\delta_{\mathrm{s}} / d_{50}=\beta \theta_{\max }$, with calibration factor $\beta=10.6$. For velocity-skewed oscillatory flow, the phase lag parameter for the positive half-cycle is greater than that for the negative half-cycle because (a) $\delta_{\mathrm{s}}$ is greater due to higher Shields and (b) flow deceleration time is shorter $\left(\left(T_{\mathrm{c}}-T_{\mathrm{cu}}\right)<\left(T_{\mathrm{t}}-T_{\mathrm{tu}}\right)\right)$; the result is a greater proportion of positive half-cycle-entrained sand being carried into the following negative half-cycle than negative halfcycle sand being carried into the following positive half-cycle, as seen in Figure 5. Other half-cycle predictors use a phase lag parameter similar in concept to that of the santoss predictor for sheet flow conditions [Camenen and Larson, 2006; Silva et al., 2006]. For net transport in sinusoidal flow with superimposed current, Dohmen-Janssen et al. [2002] applied a "reduction factor", $r$, to Ribberink's quasi-steady predictor (Equation (4)) with $r$ depending on a version of the phase lag parameter and the ratio between the current velocity and the amplitude of the sinusoidal velocity. There are other predictors for sheet-flow transport [Soulsby and Damgaard, 2005; Nielsen, 2006; Wang, 2007; Gonzalez-Rodriguez and Madsen, 2007, Suntoyo et al. 2008] which do not account for phase lag effects and so are limited to relatively coarse sand conditions.

\section{ACCELERATION EFFECTS}

For oscillatory flow, bed shear stress is usually related to the square of the flow velocity via a friction factor dependent on relative roughness $a / k_{\mathrm{s}}$, where $a=\sqrt{2} u_{\mathrm{rms}} T / 2 \pi$ and $T$ is flow period. On this basis, net transport in acceleration-skewed (sawtooth-type) flow should be zero because of the symmetry in velocity (and, therefore, bed shear stress) between the positive and negative flow half-cycles. However, experiments by King [1991] and, later, full-cycle acceleration-skewed oscillatory flow experiments by Watanabe and Sato [2004] and at larger scale by Van $\operatorname{der} A$ et al. [2010] showed that acceleration asymmetry can generate significant net sand transport in the direction of highest acceleration, and increasing net transport with increasing acceleration skewness. The net transport arises from an asymmetry in bed shear stress caused by the asymmetry in acceleration: higher flow acceleration generates higher bed shear stress because the boundary layer has less time to develop before peak velocity is reached.

The effects of acceleration skewness on oscillatory boundary layer dynamics and bed shear stress were studied in detail by Van der A et al. [2011]. An example result is presented in Figure 6, which shows the asymmetry in bed shear stress between the positive and negative flow half-cycles for oscillatory flow with high acceleration skewness alongside the symmetrical bed shear stress behaviour of the corresponding sinusoidal flow. Van $\operatorname{der} A$ et al. report values as high as 1.8 for the ratio of peak positive to peak negative bed shear stress. A number of practical predictors account for this acceleration effect on bed shear stress, including Watanabe and Sato [2004], Silva et al. [2006], Nielsen [2006] and Gonzalez-Rodriguez and Madsen [2007], Camenen and Larson [2007], and Suntoyo et al. [2008].

The santoss predictor [Van der A et al., subm.] is driven by bed shears stress, with bed shear stress evaluated separately for each half-cycle. In this predictor the acceleration skewness effect on the bed shear stress is accounted for by adjusting the relative roughness to $\left(2 T_{i \mathrm{u}} / T_{i}\right)^{\alpha} a / k_{\mathrm{s}}$, where, as before, the $T$ 's are as indicated in Figure 1 with subscript $i$ being $c$ or $u$ for wave crest or trough respectively, and $\alpha$ is a calibration parameter. So, for example, a forward-leaning crest half-cycle has $\left(2 T_{\mathrm{cu}} / T_{\mathrm{c}}\right)^{\alpha}<1$, leading to higher friction factor and bed shear stress compared to the equivalent sinusoidal flow for which $\left(2 T_{\mathrm{cu}} / T_{\mathrm{c}}\right)^{\alpha}=1$. This

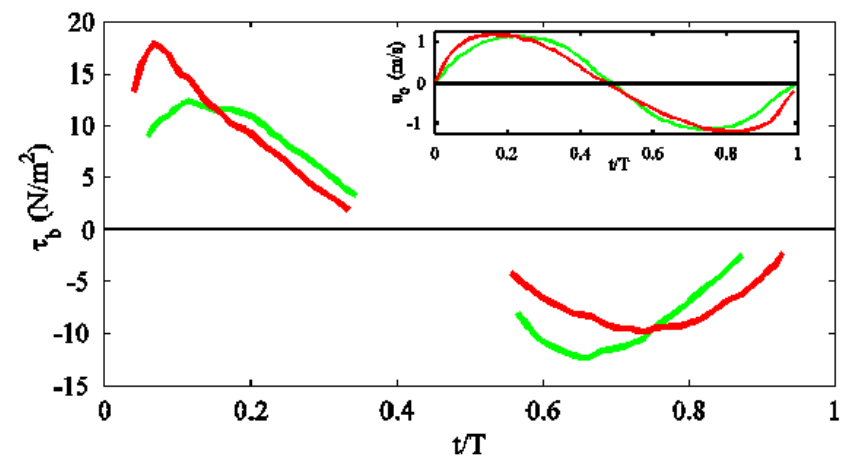

Figure 6. Measured bed shear stress time-series for sinusoidal (green) and acceleration-skewed (red) oscillatory flows over fixed rough bed. $\left(T=6 s, u_{0 \max } \approx 1.1 \mathrm{~m} / \mathrm{s}, k_{s}=14 \mathrm{~mm}\right)$. 
approach of effectively adjusting the relative roughness through the effective $a$ is similar to that used by Gonzalez-Rodriguez and Madsen [2007] in their approach to account for acceleration in the bed shear stress.

As for velocity-skewed flow, phase lag effects become significant for acceleration-skewed flow when the sand is fine and the flow period is relatively short. However, in contrast to velocity-skewed flow, for which phase lag effect augments the negative transport, phase lag tends to augment the positive transport in the case of acceleration-skewed flow (for a forwardleaning wave). This is because the deceleration time for the negative half-cycle $\left(T-T_{\mathrm{tu}}\right)$ is less than that for the positive halfcycle $\left(T_{\mathrm{c}}-T_{\mathrm{cu}}\right)$, so that sand entrained in the negative half-cycle has less time to settle before the flow reverses. The effect is seen in Figure 5(c), which shows time-varying erosion depth and sheetflow layer thickness for a fine sand in a pure acceleration-skewed flow: erosion depth and sheet-flow layer thickness are greater at the negative-positive flow reversal than at the positive-negative flow reversal; a greater proportion of sand entrained during the negative half-cycle is carried into the following positive half-cycle than is carried from the positive half-cycle into the following negative half-cycle. For half-cycle-based practical predictors, the effect is captured via the phase lag parameter as previously defined.

\section{PROGRESSIVE WAVE EFFECTS}

Of the 226 large-scale laboratory conditions for which net transport has been measured, 11 correspond to progressive, nonbreaking waves in a large wave flume; all other cases are for oscillatory flows in large oscillatory flow tunnels. Figure 7 shows progressive wave net transport rates plotted against $\sqrt{2} u_{\text {rms }}$ for sands with $0.21 \leq d_{50} \leq 0.27 \mathrm{~mm}$, with the data coming from two independent experimental series [Dohmen-Janssen and Hanes, 2002; Schretlen et al., 2011]. Also shown are measured net transport rates from large-scale oscillatory flow tunnel experiments with near-bed velocity-skewed flows and sand size similar to those of the progressive wave cases. The results indicate that net transport rates for medium sand under progressive waves are higher than transport rates in "similar" oscillatory flows. Moreover, Schretlen et al. [2010] report that net transport for fine sand $\left(d_{50} \approx 0.15 \mathrm{~mm}\right)$ is positive under progressive waves, in contrast to the negative net transport measured for fine sand in strong velocity-skewed oscillatory flows (as seen, for example, in Figure 4). The indication is that boundary layer streaming and/or horizontal advection of sand contributes significantly to the net transport. Unfortunately, unlike for tunnel flows, detailed boundary layer measurements with which to better understand the streaming and the boundary layer shear stresses are lacking for large-scale waves over fixed rough beds. However, Kranenburg et al. [2011] used a numerical model that can simulate both wave flume and oscillating tunnel flows to show that the wave-induced streaming does indeed contribute to the higher net transport rates seen under progressive waves. With the exceptions of Nielsen [2006], Van Rijn [2007] and Van der A et al. [subm.], existing practical predictors make no distinction between oscillatory nearbed flow as occurs in tunnels and oscillatory near-bed flow generated by progressive surface waves. Both Nielsen [2006] and

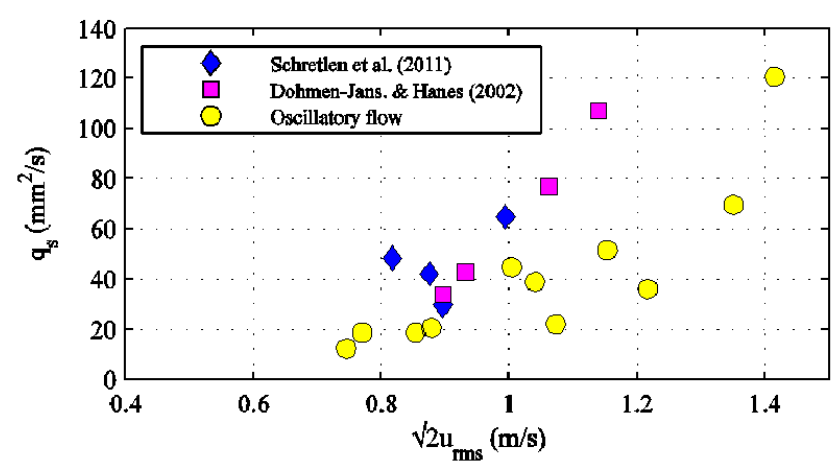

Figure 7. Measured sand transport rates from progressive surface waves [Dohmen-Janssen and Hanes, 2002; Schretlen et al. 2011] and oscillatory flow tunnel experiments with "similar" flow and sand conditions $\left(0.21 \leq d_{50} \leq 0.27 \mathrm{~mm} ; 0.57<R<0.72 ; 5 s<T<\right.$ $10 s)$.

Van $\operatorname{der} A$ et al. [subm.] account for the effect of wave-related positive streaming by adding a positive wave Reynolds stress, $\tau_{\mathrm{wRe}}$, in the following manner:

$$
\theta_{i}=\frac{\frac{1}{2} f_{\mathrm{w}}\left|u_{i}\right| u_{i}+\tau_{w \mathrm{Re}}}{(s-1) g d_{50}}
$$

where $\tau_{\mathrm{wRe}}$ is evaluated using

$$
\tau_{\mathrm{wRe}}=\frac{2}{3 \pi} \rho f_{\mathrm{w}} \frac{u^{3}}{c}
$$

in which $f_{\mathrm{w}}$ is friction factor, $s$ is sediment specific gravity, $g$ is acceleration due to gravity, $\rho$ is water density and $c$ is wave speed.

Kranenburg et al. [2011] have also shown that other waverelated processes are at work, which impact particularly on the net transport of fine sand: (i) vertical advection of sand by vertical orbital velocities, which causes sand to settle faster at the end of the onshore flow and settle slower at the end of the offshore flow; and (ii) higher concentration peaks and enhanced flux at times of maximum positive velocity caused by horizontal gradients in the horizontal sediment flux. In the santoss predictor [Van der A et al., subm.], account is taken of (i) by simple adjustment of the sand settling velocity under the wave crest, $w_{\mathrm{sc}}$, and wave trough, $w_{\mathrm{st}}$, as follows:

$$
w_{s c}=w_{s}+v_{r} \quad ; \quad w_{s t}=\max \left[\left(w_{s}-v_{r}\right), 0\right]
$$

where $w_{\mathrm{s}}$ is settling velocity in still water and $v$ is the amplitude of orbital vertical velocity at reference height $r$ above the bed, with $r$ equal to ripple height for rippled bed and equal to sheet-flow layer thickness for sheet flow. In the same predictor, account is taken of (ii) through a correction to the phase lag parameter $P_{\mathrm{i}}$ (Equation (3)) that increases $P_{\mathrm{i}}$ under the wave crest and reduces $P_{\mathrm{i}}$ under the trough; see Van der A et al. [subm.] for details.

\section{CONCLUSION}

Since the first NCK-Days 20 years ago, a significant body of large-scale laboratory experiments on wave-driven sand transport has been conducted in oscillatory flow tunnels and very large wave flumes. The experiments have yielded measures of net sand transport rates for a wide range of flow and sand conditions and have provided insights that have informed the development of 
practical predictors. Consequently, the most developed predictors now account for the ripple and sheet flow regimes, unsteady phase lag effects, wave shape effects including flow acceleration, and the differences between oscillatory flow as occurs in tunnels and oscillatory flow generated by progressive surface waves. The paper has presented a brief overview of the experiments along with indications of how insights and quantitative results have been used to develop the predictors.

Although the body of experimental work is substantial, it is the case that some hydrodynamic conditions have received more attention than others. For example, despite recent significant work, experiments involving progressive surface waves are still relatively few and more experimental research is needed with fixed and mobile beds under large-scale surface waves to better measure the fundamental processes. However, the most significant shortfall in the coverage of experiments to date is arguably the fact that they are limited to non-breaking wave conditions, which of course means that the attendant predictors are limited to nonbreaking waves. For this reason a primary objective of further research in this area must be to conduct large-scale experiments with breaking waves, measuring net transport rates and fundamental processes as before, and to look to extend practical predictors to accommodate breaking wave conditions.

\section{ACKNOWLEDGEMENT}

Much of the Aberdeen research reported in this paper was conducted in collaboration with Jan Ribberink and colleagues at the University of Twente over the past number of years. The "santoss" work mentioned in the paper refers to our recent collaboration on the SANTOSS project ('SANd Transport in OScillatory flows in the Sheet-flow regime') funded by the UK's EPSRC (GR/T28089/01) and STW in The Netherlands (TCB.6586).

\section{REFERENCES}

Van der A, D. A., T. O'Donoghue, and J. S. Ribberink (2010), Measurements of sheet flow sediment transport in accelerationskewed oscillatory flow and comparison with practical formulations. Coastal Engineering, 57(3), doi: 10.1016/ j.coastaleng.2009.11.006.

Van der A, D. A., T. O'Donoghue, A. G. Davies, and J. S. Ribberink (2011), Experimental study of the turbulent boundary layer in acceleration-skewed oscillatory flow. Journal of Fluid Mechanics, 684, 251-283, doi:10.1017/jfm.2011.300.

Van der A, D. A., J. S. Ribberink, J. J. van der Werf, T. O'Donoghue, R. H. Buijsrogge, and W. M. Kranenburg (submitted), New practical sand transport formula for nonbreaking waves and currents. Coastal Engineering.

Ahmed, A. S. M., and S. Sato (2001), Investigation of bottom boundary layer dynamics of movable bed by using enhanced PIV technique, Coastal Engineering, 43(4), 239-258. doi:10.1142/S0578563401000360.

Camenen, B. and M. Larson (2006), Phase-lag effects in sheet flow transport, Coastal Engineering, 53(5-6), 531-542, doi: 10.1016/j.coastaleng.2005.12.003.
Camenen, B., M. Larson (2007), A total load formula for the nearshore. Proceeding Coastal Sediments '07. ASCE, New Orleans, Louisiana, USA.

Cataña-Lopera, Y. A., and M. H. Garcia (2006), Geometry and migration characteristics of bedforms under waves and currents Part 2: Ripples superimposed on sandwaves, Coastal Engineering, 53(9), 767-780, doi: /10.1016/j.coastaleng.2006. 03.007.

Dohmen-Janssen, C. M., D. M. Hanes (2002), Sheet flow dynamics under monochromatic nonbreaking waves. Journal of Geophysical Research, 107(C10), 3149, doi:10.1029/2001 JC001045.

Dohmen-Janssen, C. M., D. Kroekenstoel, W.N. Hassan, and J. S. Ribberink (2002), Phase lags in oscillatory sheet flow: experiments and bed load modelling, Coastal Engineering, 46(1), 61-87, doi:10.1016/S0378-3839(02)00056-X.

Drake, T. G., and J. Calantoni (2001), Discrete particle model for sheet flow sediment transport in the nearshore. Journal of Geophysical Research, 106(C9), 19,859-19,868, doi: 10.1029/ 2000JC000611.

Dibajnia, M., and A. Watanabe (1992), Sheet flow under non linear waves and currents. Proceedings $23^{\text {rd }}$ International Conference on Coastal Engineering, ASCE, 2015-2028.

Dibajnia, M., and A. Watanabe (1996), A transport rate formula for mixed sands. Proceedings $25^{\text {th }}$ International Conference on Coastal Engineering, ASCE, 3791-3804.

Dibajnia, M. and A. Watanabe (1998), Transport rate under irregular sheet flow conditions. Coastal Engineering, 35(3), 167-183, doi:10.1016/S0378-3839(98)00034-9.

Dumas, S., R. W. C. Arnott, and J. B. Southard (2005), Experiments on oscillatory-flow and combined-flow bed forms: Implications for interpreting parts of the shallow-marine sedimentary record. Journal of Sedimentary Research, 75(3), 501-513, doi:10.2110/jsr.2005.039.

Gonzalez-Rodriguez, D., and O. S. Madsen (2007), Seabed shear stress and bedload transport due to asymmetric and skewed waves. Coastal Engineering, 54(12), 914-929, doi:10.1016/ j.coastaleng.2007.06.004.

King, D. B. (1991), Studies in oscillatory flow bedload sediment transport. PhD thesis, University of California, Sand Diego.

Kranenburg, W. M., J. S. Ribberink, R. E. Uittenbogaard (2011), Sand transport by surface waves: can streaming explain onshore transport? Proceedings of the International Conference on Coastal Engineering, 32(2010), Paper\#: sediment.11.

Nielsen, P. (2006), Sheet flow sediment transport under waves with acceleration skewness and boundary layer streaming. Coastal Engineering, 53(9), 749-758, doi: 10.1016/j.coastaleng. 2006.03.006.

O'Donoghue, T., and G. S. Clubb (2001), Sand ripples generated by regular oscillatory flow, Coastal Engineering. 44(2), 101115, doi: 10.1016/S0378-3839(01)00025-4.

O'Donoghue, T., J. S. Doucette, J. J. van der Werf, and J. S. Ribberink (2006), The dimensions of sand ripples in full-scale oscillatory flows, Coastal Engineering, 53(12), 997-1012, doi: 10.1016/j.coastaleng.2006.06.008.

O'Hara Murray, R. R., P. D. Thorne, D. M. Hodgson (2011), Intrawave observations of sediment entrainment processes 
above sand ripples under irregular waves, Journal Geophysical Research, 116(C01001), doi:10.1029/2010JC006216.

Pedocchi, F., and M. H. Garcia (2009), Ripple morphology under oscillatory flow: 2. Experiments, Journal Geophysical Research, 114(C12015), doi:10.1029/2009JC005356.

Ribberink, J. S., and A. A. Al-Salem (1994), Sediment transport in oscillatory boundary layers in cases of rippled beds and sheet flow. Journal of Geophysical Research, 99(C6), 12,707-12,727. doi:10.1029/94JC00380.

Ribberink, J. S., and A. A. Al-Salem (1995), Sheet flow and suspension of sand in oscillatory boundary layers. Coastal Engineering, 25(3-4), 205-225, doi: 10.1016/0378-3839(95) 00003-T.

Ribberink, J. S. (1998), Bed-load transport for steady flows and unsteady oscillatory flows, Coastal Engineering, 34(1-2), 59-82, doi:10.1016/S0378-3839(98)00013-1.

Ribberink, J.S., J.J. van der Werf, T. O'Donoghue, and W.N.M. Hassan (2008), Sand motion induced by oscillatory flow: sheet flow and vortex ripples. Journal of Turbulence, 9(20), 1-33, doi: $10.1080 / 14685240802220009$.

Van Rijn, L. C. (2007), Unified view of sediment transport by currents and waves. I: Initiation of motion, bed roughness, and bed-load transport. Journal of Hydraulic Research, 133(6), 649667, doi: 10.1061/(ASCE)0733-9429(2007)133:6(649).

Schretlen, J. L. M., J. S. Ribberink, T. O'Donoghue (2011), Boundary layer flow and sand transport under full scale surface waves. Proceedings of the International Conference on Coastal Engineering, 32(2010), Paper\#: sediment.4.

Sleath, J. F. A., and S. Wallbridge (2002), Pickup from rippled beds in oscillatory flow. Journal of Waterway, Port, Coastal and Ocean Engineering. 128(6), 228-237, doi: 10.1061/(ASCE) 0733-950X(2002)128:6(228).

Soulsby, R. L., and J. S. Damgaard (2005), Bedload sediment transport in coastal waters. Coastal Engineering, 52(8), 673 689, doi:10.1016/j.coastaleng.2005.04.003.

Suntoyo, H. Tanaka, and A. Sana (2008), Characteristics of turbulent boundary layers over a rough bed under saw-tooth waves and its application to sediment transport. Coastal Engineering, 55(12), doi: 10.1016/j.coastaleng.2008.04.007.

Silva, P. A., A. Temperville, and F. Seabra Santos (2006), Sand transport under combined current and wave conditions: A semiunsteady, practical model. Coastal Engineering, 53(11), 897913, doi:10.1016/j.coastaleng.2006.06.010.

Thorne, P., A. G. Davies, and J. J. Williams (2003), Measurements of near-bed intra-wave sediment entrainment above vortex ripples. Geophysical Research Letters, 30(20), doi:10.1029/2003GL018427.

Wang, Y.-H. (2007), Formula for predicting bedload transport rate in oscillatory sheet flows, Coastal Engineering, 54(8), 594-601, doi:10.1016/j.coastaleng.2006.12.003.

Watanabe, A., S. Sato, (2004), A sheet-flow transport rate formula for asymmetric forward-leaning waves and currents. Proceedings 29th International Conference on Coastal Engineering. World Scientific, 1703-1714.

Van der Werf, J. J., J. S. Ribberink, T. O'Donoghue and J.S. Doucette, (2006), Modelling and measurement of sand transport processes over full-scale ripples in oscillatory flow. Coastal Engineering, 53(x), 657-673, doi :10.1016/j.coastaleng. 2006.02.002.

Van der Werf, J. J., J. J. L. M. Schretlen, J. S. Ribberink, and T. O'Donoghue (2009), Database of full-scale laboratory experiments on wave-driven sand transport processes. Coastal Engineering, 56(7), 726-732, doi: 10.1016/j.coastaleng.2009. 01.008 .

Van der Werf, J. J., J. S. Ribberink, and T. O'Donoghue (2007), Development of a new practical model for sand transport induced by non-breaking waves and currents. Proceedings Coastal Dynamics '07, ASCE, 1-14.

Williams, J.J., P. S. Bell, P. D. Thorne, N. Metje, and L. E. Coates (2004), Measurement and prediction of wave-generated suborbital ripples, Journal Geophysical Research, 109(C02004), doi: $10.1029 / 2003$ JC001882. 